\title{
Validation of Montreal Cognitive Assessment, MoCA, Alternate French Versions
}

\author{
Ziad Samir Nasreddine, Bhavini Bhovan Patel
}

\begin{abstract}
Objective background: The Montreal Cognitive Assessment (MoCA) is a questionnaire that has been developed to help physicians around the world diagnose a patient's cognitive ability. Available in multiple languages and for use in multiple countries worldwide, the goal of this study was to validate the alternate versions 2 and 3 of the French MoCA test to assist physicians in the detection of mild cognitive impairment (MCI), while decreasing the learning effect upon frequent testing. Methods: A validation study was conducted at the MoCA Clinic and Institute in Québec, Canada. The subject population consisted of 25 patients diagnosed with MCI meeting Petersen criteria and 25 healthy subjects serving as the normal control (NC) group. Three MoCA test versions were administered in the French language in random order within one session. Scores obtained in all three versions in MCI and NC groups were assessed for reliability and consistency from one version to the next. Results: On average, scores obtained in each subject group (MCI and NC) fell within their corresponding diagnostic ranges (score above 26 points for NC patients versus scores below 26 points for MCI patients). Difference in scores observed between the original French MoCA version and the two alternate versions in each subject cohort were minimal and not considered clinically significant. Conclusions: All three test versions of the French MoCA are considered equivalent in diagnostic reliability and consistency and contribute to decreasing the potential learning effect when patients are required to repeat the test frequently.
\end{abstract}

RÉSUMÉ: Validation des deux versions de remplacement du test français d'évaluation cognitive de Montréal. Contexte: Le test d'évaluation cognitive de Montréal (Montreal Cognitive Assessment ou MoCA ci-après) est un questionnaire qui a été conçu pour aider les médecins à déterminer la capacité cognitive d'un patient. Ce questionnaire est disponible en plusieurs langues et peut ainsi être utilisé dans de nombreux pays. Le but de cette étude est donc de valider les versions de remplacement 2 et 3 du MoCA en français, notamment la diminution de l'effet d'apprentissage associé au fait de passer de fréquents tests, afin d'aider les médecins dans le dépistage de troubles cognitifs légers (TCL). Méthodes: Une étude de validation a été menée au Québec (Canada) à la clinique et à l'institut du MoCA. L'échantillon étudié comptait 25 patients chez lesquels on avait diagnostiqué un TCL suivant les critères de Petersen ainsi que 25 sujets en santé inclus dans un groupe témoin (GT). Dans un ordre défini au hasard et au cours d'une même séance, les participants ont été soumis à trois versions françaises du test MoCA. Nous avons ensuite évalué la cohérence et la fiabilité des scores obtenus dans le groupe de patients atteints d'un TCL et le GT pour ces trois versions du MoCA. Résultats: En moyenne, les scores obtenus dans chacun des groupes (TCL et GT) sont demeurés fidèles à l'étendue des valeurs diagnostiques, à savoir des scores dépassant les 26 points pour les patients du GT versus des scores inférieurs à 26 points pour les patients atteint d'un TCL. Les différences de scores observées entre la première version du test MoCA et les deux versions de remplacement, et ce, dans chacun des groupes, se sont révélées minimes et n'ont pas été jugées importantes sur le plan clinique. Conclusions: Les trois versions françaises du test MoCA peuvent être considérées équivalentes en ce qui concerne leur cohérence et leur fiabilité en plus de contribuer à diminuer un potentiel effet d'apprentissage dans le cas où des patients doivent repasser ce test à maintes reprises.

Keywords: Alzheimer's, Cognitive impairment, Clinical neurosciences

doi:10.1017/cjn.2016.273

Can J Neurol Sci. 2016; 43: 665-671

Mild cognitive impairment (MCI) is a transition state between normal aging and dementia. MCI diagnosis is most often determined by the presence or absence of memory impairment, the ability to continue general cognitive and functional activities, and the absence of diagnosed dementia. ${ }^{1-3}$

The MoCA, Montreal Cognitive Assessment, is 10-minute, one-page, 30-point cognitive screening test ${ }^{4}$ used around the world to help detect MCI.

Although MoCA was designed as a diagnostic screening tool, clinicians have increasingly used it on longitudinal assessments. Repeated measures may lead to a practice effect and decrease the test validity. The development of reliable alternate versions would counteract these inconveniences.
In 2012, Ana Costa et al developed two alternate forms for the German MoCA test with the goal of determining the reliability of the alternate forms for longitudinal assessments. ${ }^{5}$ The original and one of two alternate forms were administered to MCI, Alzheimer's disease, and a control group of patients within

From the MoCA Clinic and Institute, Greenfield Park, Québec, Canada (ZSN); University of Sherbrooke, Sherbrooke, Québec, Canada (ZSN); McGill University, Montreal, Québec, Canada (ZSN, BBP).

Received July 28, 2015. Final Revisions Submitted March 5, 2016. Date of ACCEPTENCE APRIL 12, 2016.

Correspondence to: Ziad Nasreddine, MoCA Clinic and Institute, 4892 Taschereau Blvd, Greenfield Park, Québec, Canada, J4V 2J2. Email: ziad.nasreddine@ cedra.ca 
a 60-minute interval. A strong correlation between the alternate forms and the original MoCA was observed, indicating that all three forms could be used with reliability and alternatively for cognitive assessment.

The purpose of this study is to validate the alternate versions 2 and 3 of the French MoCA test to assist physicians in the detection of $\mathrm{MCI}$, while decreasing the learning effect upon repeated testing.

\section{Methods}

\section{Participants}

Twenty-five subjects with amnestic MCI who met the Petersen criteria, ${ }^{6}$ who underwent a recent (less than 6 months) confirmation by a neuropsychological assessment (with a minimum of 1.5 standard deviations below normal on the Rey Auditory Verbal Learning Test) were recruited at the MoCA Clinic and Institute in Québec, Canada, to form the MCI group. Subjects were aged 55 years or older (average age, 72.69); both males and females were included. Subjects had to be fluent in French.

An additional 25 patients were recruited to form the normal control (NC) group. Subjects in this group considered themselves healthy, 65 years of age and older (average age, 72.88), had 5 years or more of formal education, functioned independently in the community, and also had to be fluent in French. Healthy controls did not undergo neuropsychological assessment, but were not considered depressed according to the Geriatric Depression scale and did not exhibit significant cognitive complaints on Subjective Memory Scale (SMS). See Appendix 1 for inclusion and exclusion criteria.

All subjects signed a written informed consent. The study was approved by an independent ethics committee.

\section{Procedure}

Eligible subjects that were recruited were tested with MoCA version 1 (original MoCA version), version 2, and version 3 within the same session. The counterbalanced method was used to have a balanced order of administration of versions 1,2 , and 3 that are presented equally in terms of the sequence in positions 1,2 , or 3 for each group of subject group. All three versions were administered back to back within 30 minutes (10 minutes per test). The test was administered to the subjects in each group (MCI and NC) by the same examiner; however, a different examiner was used to correct all the subjects' tests.

The test was administered to patients as they were seated comfortably in a quiet room in an environment that would elicit compliant participation and cooperation. All tests and medical evaluations were conducted at the MoCA Clinic and Institute.

\section{MoCA Test and Alternate Versions}

Alternate versions' development consisted of replacing all elements of the original MoCA test with equivalent elements that respect complexity, level of difficulty, administration and scoring time, linguistic frequency, cultural compatibility, cognitive domain specificity, and ability to discriminate between healthy controls and patients with MCI as well as the original version in terms of sensitivity and specificity and cutoff scores.

New test items had to be very close to the original version because they were already shown to discriminate between normal controls and subjects with MCI. They also had to be different to prevent a learning effect. All new test elements for the new versions were chosen by one author $(\mathrm{ZN})$ by testing the items on the memory clinic staff and making changes while preserving the concept, complexity, ease of administration/rating, and category of information compared with the original version.

The item-by-item changes follow.

- Trail: Alternating between numbers and letters; only the spatial location of the numbers and letters were changed.

- Cube: a different three-dimensional element was chosen with a similar time to complete the task.

- Clock: The time was changed to 9:10 (version 7.2) from 11:10; the subject may choose to put the minute hand on the number 10 , thus preserving the abstraction/executive function element as well as the same visuospatial elements. The same concept was used for version 3 with the time at 10:05.

- Animal naming: lion, rhino, and camel were replaced with a similar complex of and frequently known animals (version 7.2: snake, elephant, and crocodile; version 7.3: horse, tiger, and duck).

- Memory: Five words were chosen to respect the category, frequency, and length of the words in version 1; for example, face was replaced by hand (in French: main) in version 7.2, and leg (in French: jambe) in version 7.3.

- Attention: digit span: the forward and backward numbers were shuffled only.

- Letter A: not changed because the learning effect was not considered to be possible.

- Serial 7: the same concept was kept by subtracting from a round number, starting at 70 for version 2 and 60 for version 3 , instead of 100.

- Sentence repetition: the number of words, grammatical complexity, and understandability were respected.

- Letter fluency: Equally frequent letters were chosen by using their frequency in the dictionary and testing them on the clinic staff.

- Abstraction: The same level of difficulty and frequency of the elements was respected, with one easier task and one harder.

- Orientation: Not changed because it already varies from one test to the next.

In a preliminary study, where alternate versions 2 and 3 in subjects speaking French were administered to MCI $(n=25)$, AD $(n=28)$, and NC $(n=33)$ patients, results revealed that these versions were slightly more difficult than the original MoCA version. As a result, changes to versions 2 and 3 were made that included replacing a few components in the MoCA 2 and MoCA 3 tests (Tables 1 and 2). See www.mocatest.org for the original French MoCA version 7.1 and the two alternate MoCA 7.2 and 7.3 versions used to test subjects in this study.

\section{Statistical Analysis}

Sample sizes were based on practical possibility of recruiting patients as well as being based on prior experience with the MoCA validation study. ${ }^{4}$ A minimum sample size of 25 subjects per group was chosen to provide a minimum of $80 \%$ power (this is considered to be within the reasonable norms of a minimum of $80 \%$ to $90 \%$ ), which is able to detect an effect size of 0.6 points in this study and is considered moderate to high according to Cohen. Clinically, a difference of 2 points is considered significant on a 30-point test according to the author's (ZN) clinical experience with this test. 
Table 1: Summary of the changes made from the older French MoCA version 2

\begin{tabular}{l|l|l}
\hline French MoCA 2 & Initial & Changed to \\
\hline Animal 1 & Goat & Snake \\
\hline Sentence 1 & $\begin{array}{l}\text { La personne accusée de } \\
\text { vol a été déclarée coupable } \\
\text { par le juge }\end{array}$ & $\begin{array}{l}\text { Le voleur de l'auto grise a été } \\
\text { arrêté par la police }\end{array}$ \\
\hline Word 2 & Soie & Nylon \\
\hline Word 3 & Temple & Parc \\
\hline Word 5 & Gris & Jaune \\
\hline Abstraction 1 & Sculpture-Peinture & Lit-Table \\
\hline
\end{tabular}

The "Initial" column refers to what the alternate version 2 for the French test contained. The "Changed to" column refers to the changes made on the test for specific items from the "initial" version 2 test. The test containing the changes is now the French alternate MoCA 2 version used for diagnostic purposes.

Validation and comparison assessments were conducted by performing $t$ tests and determining intraclass correlation coefficients among the three MoCA test versions as well as the MCI versus NC study subject groups.

Intraclass correlation coefficients (ICCs) are generally calculated to understand consistency or conformity between two or more quantitative measurements. ${ }^{7}$ Given that all subjects were given all MoCA versions by one administrator, ICCs were calculated, again using SPSS, to assess the reliability and consistency of score measurements by a single test administrator during the multiple test distributions. Scores of the three possible combinations of alternate MoCA versions were compared in both subject populations ( $\mathrm{NC}$ and $\mathrm{MCI}$ ). A single measure ICC value that falls between the upper and lower bound of the $95 \%$ confidence interval indicates consistency of measurements from one test score to the next by the same test administrator. Although the SPSS-generated test results also provide an average measure ICC, this value is not discussed in this case because the value is an estimate that is computed assuming the interaction effect is absent.

Moreover, two-tailed paired sample tests using SPSS was also performed among the different MoCA versions to assess whether the difference of mean scores generated by the two subject groups

Table 2: Summary of the changes made from the older French MoCA version 3

\begin{tabular}{l|l|l}
\hline French MoCA 3 & Initial & Changed to \\
\hline Drawing & Three-dimensional house & Three-dimensional bed \\
\hline Animal 1 & Eagle & Horse \\
\hline Animal 2 & Gorilla & Tiger \\
\hline Word 1 & Nez & Jambe \\
\hline Word 2 & Laine & Coton \\
\hline
\end{tabular}

The "Initial" column refers to what the alternate version 3 for the French test contained. The "Changed to" column refers to the changes made on the test for specific items from the "initial" version 3 test. The test containing the changes is now the French alternate MoCA 3 version used for diagnostic purposes.
Table 3: Mean score for NC and MCI population groups who were tested with all three MoCA test versions

\begin{tabular}{l|l|l|l|c|c}
\hline \multicolumn{2}{l|}{ Group } & N & Mean & $\begin{array}{c}\text { Standard } \\
\text { deviation }\end{array}$ & $\begin{array}{c}\text { Standard error of the } \\
\text { mean }\end{array}$ \\
\hline $\begin{array}{c}\text { Score } \\
\text { MoCA 1 }\end{array}$ & NC & 25 & 26.48 & 2.931 & 0.586 \\
\cline { 2 - 6 } & MCI & 25 & 21.24 & 3.574 & 0.715 \\
\hline \multirow{2}{*}{$\begin{array}{c}\text { Score } \\
\text { MoCA 2 }\end{array}$} & NC & 25 & 25.96 & 2.879 & 0.576 \\
\cline { 2 - 6 } & MCI & 25 & 20.48 & 3.216 & 0.643 \\
\hline \multirow{2}{*}{$\begin{array}{c}\text { Score } \\
\text { MoCA 3 }\end{array}$} & NC & 25 & 25.80 & 3.014 & 0.603 \\
\cline { 2 - 6 } & MCI & 25 & 21.44 & 3.216 & 0.643 \\
\hline
\end{tabular}

Mean score was generated from each subject group, where $\mathrm{N}=25$.

from one MoCA version to the next was of significance or could be disregarded. In other words, the paired $t$ test was used to assess whether the mean scores of the alternate versions agreed with each other, on average.

A receiver operating characteristics plot (ROC curve) was also generated to visualize accuracy for MCI detection amongst the three MoCA test versions.

\section{RESUltS AND DiscuSSION}

Mean average score for NC subjects from MoCA version 1 varied by -0.52 to -0.68 points with a variation in standard error of -0.1 to +0.017 points in comparison to MoCA versions 2 and 3 . Whereas, MCI subjects scored an average of 0.76 to 0.2 points lower on MoCA versions 2 and 3, relative to MoCA 1. Similarly, standard error for the two alternate versions was also lower in comparison to MoCA version 1 ( \pm 0.072 points lower) (Table 3 ).

Given the scores presented in Table 3, it can be seen that in all three MoCA versions, the MCI group was consistently below 26 points, which is the cognitively impaired range (MoCA 1 mean score $=21.24$; MoCA 2 mean score $=20.48$; MoCA 3 mean score $=21.44$ ). On the other hand, in the NC subject group, all three MoCA versions demonstrated mean scores ranging from 25.80 to 26.48 , which is in the normal range.

Table 4: Correlation coefficients and $p$ values for mean average MoCA test scores in three possible combination pairs in the NC and MCI subject groups

\begin{tabular}{|c|c|c|c|c|c|}
\hline \multicolumn{3}{|c|}{ Group } & \multirow{2}{*}{$\frac{\mathbf{N}}{25}$} & \multirow{2}{*}{$\begin{array}{c}\boldsymbol{r} \\
0.820\end{array}$} & \multirow{2}{*}{$\frac{\mathbf{p}}{0.000}$} \\
\hline MCI & Pair 1 & Score_MoCA 1 and Score_MoCA 2 & & & \\
\hline & Pair 2 & Score_MoCA 1 and Score_MoCA 3 & 25 & 0.842 & 0.000 \\
\hline & Pair 3 & Score_MoCA 2 and Score_MoCA 3 & 25 & 0.813 & 0.000 \\
\hline \multirow[t]{3}{*}{$\mathrm{NC}$} & Pair 1 & Score_MoCA 1 and Score_MoCA 2 & 25 & 0.827 & 0.000 \\
\hline & Pair 2 & Score_MoCA 1 and Score_MoCA 3 & 25 & 0.733 & 0.000 \\
\hline & Pair 3 & Score_MoCA 2 and Score_MoCA 3 & 25 & 0.676 & 0.000 \\
\hline
\end{tabular}

$\mathrm{N}=25$; pair $1=$ score between MoCA 1 and MoCA 2; pair $2=$ score between MoCA 1 and MoCA 3; pair $3=$ score between MoCA 2 and MoCA 3. A value of $\mathrm{p}<0.05$ is indicative of significance. A positive correlation coefficient $(r)>0.5$ is indicative of a positive linear relationship in a pair. The closer the positive $r$ gets to +1.0 , the stronger the positive linear relationship within a given pair. 
Table 5: Two-tailed paired sample $t$ test for the three possible combination pairs of MoCA versions in the NC and MCI subject groups, determined by SPSS

\begin{tabular}{|c|c|c|c|c|c|c|c|c|c|c|}
\hline & & & \multicolumn{5}{|c|}{ Paired differences } & \multirow[b]{3}{*}{$t$ value } & \multirow[b]{3}{*}{ df } & \multirow[b]{3}{*}{ Significance (p) (2-tailed) } \\
\hline & & & \multirow[b]{2}{*}{ Mean } & \multirow[b]{2}{*}{ SD } & \multirow[b]{2}{*}{ SEM } & \multicolumn{2}{|c|}{$95 \%$ CI of the difference } & & & \\
\hline & & & & & & Lower & Upper & & & \\
\hline \multirow[t]{3}{*}{ MCI } & Pair 1 & Score_MoCA 1 - Score_MoCa2 & 0.760 & 2.067 & 0.413 & -0.093 & 1.613 & 1.838 & 24 & 0.078 \\
\hline & Pair 2 & Score_MoCA 1 - Score_MoCA 3 & -0.200 & 1.936 & 0.387 & -0.999 & 0.599 & -0.516 & 24 & 0.610 \\
\hline & Pair 3 & Score_MoCA 2 - Score_MoCA 3 & -0.960 & 1.968 & 0.394 & -1.772 & -0.148 & -2.439 & 24 & 0.023 \\
\hline \multirow[t]{3}{*}{$\mathrm{NC}$} & Pair 1 & Score_MoCA 1 - Score_MoCa2 & 0.520 & 1.711 & 0.342 & -0.186 & 1.226 & 1.520 & 24 & 0.142 \\
\hline & Pair 2 & Score_MoCA 1 - Score_MoCA 3 & 0.680 & 2.174 & 0.435 & -0.217 & 1.577 & 1.564 & 24 & 0.131 \\
\hline & Pair 3 & Score_MoCA 2 - Score_MoCA 3 & 0.160 & 2.375 & 0.475 & -0.820 & 1.140 & 0.337 & 24 & 0.739 \\
\hline
\end{tabular}

$\mathrm{CI}=$ confidence interval; $\mathrm{df}=$ degrees of freedom; $\mathrm{SD}=$ standard deviation; $\mathrm{SEM}=$ standard error of the mean. Mean column depicts difference in mean average score between the alternate MoCA versions in each pair. SD and SEM provide the magnitude of the random fluctuations present within the difference of mean score; $95 \%$ CI of the difference is determined to demonstrate whether mean difference falls within the lower and upper level of confidence. Test statistic $(t)$ expresses size of the difference relative to the size of the standard error. $\mathrm{df}(\mathrm{N}=25 ; \mathrm{df}=\mathrm{N}-1=24)$, along with " $\mathrm{t}$ " allows for determining significance, where $\mathrm{p}>0.05$ implies nonsignificance. Pair 1 assesses the difference in mean average score between MoCA 1 and MoCA 2; pair 2 assesses the difference in mean average score between MoCA 1 and MoCA 3; and pair 3 assesses the difference in mean average score between MoCA 2 and MoCA 3.

Paired sample correlation test was performed in both subject groups with all three possible MoCA version pairing combinations in order to measure the strength of each test version in relation to one another. Correlation coefficients ranged from $r=0.813$ to $r=0.842$ for the MCI group. On the other hand, correlation coefficients in the $\mathrm{NC}$ group ranged from $r=0.676$ to $r=0.827$ (Table 4 ). Given that all correlation coefficients seen are positive and greater than 0.5 served as an indication that MoCA pairs in each given case shared a strong linear relationship. It was also observed that the MoCA pairs for the MCI subjects shared an even stronger linear relationship (mean correlation coefficient, $r=0.825$ ) when compared to the MoCA pairs in the NC subjects (mean correlation coefficient, $r=0.745$ ).

Where significance level was set at 0.05 , significant values for all paired combinations ranged from 0.023 to 0.739 (Table 5). The 2-tailed significant values relative to the set significance level provided a basis for determining whether the difference observed in the mean score of a given pair was important enough to give an indication as to whether each MoCA version met the adequate qualification of serving as an alternate diagnostic test version. $\boldsymbol{\rho}$-values above 0.05 were an indication of non-significance, whereas, a $\boldsymbol{\rho}$-value less 0.05 was an indication of significance in difference.

In all cases, with the exception of Pair 3 (Score_MoCA 2 Score_MoCA 3) of the MCI group, all significance values ( $\rho$ ) were greater than 0.05 (Table 5), indicating that the differences observed from the scores of one MoCA version to the next was not significant enough. In other words, the ability to correctly detect an MCI patient based on MoCA score by all three versions was still equally valid, regardless of the differences of scores observed such as between version 2 and 3. Clinically, a difference of 2 points is considered significant on a 30 point test according to the author's $(\mathrm{ZN})$ clinical experience with this test. Therefore MoCA version 2 and 3 in MCI subjects was the only pair that showed a small difference in the average total score of 0.960 , with a significant $\mathrm{p}<0.02$, was not considered clinically significant. Also Intraclass correlation coefficient comparison of MoCA 2 and MoCA 3 test scores in MCI and NC patients were comparable as

Table 6: Intraclass correlation coefficient comparison of MoCA 1 and MoCA 2 test scores in MCI and NC patients generated using SPSS

\begin{tabular}{|c|c|c|c|c|c|c|c|c|}
\hline \multicolumn{2}{|c|}{ Group } & \multirow[t]{2}{*}{$\mathrm{ICC}^{*}$} & \multicolumn{2}{|c|}{$95 \% \mathrm{CI}$} & \multicolumn{4}{|c|}{ F test with true value 0.8} \\
\hline & & & Lower bound & Upper bound & Value & df1 & df 2 & Significance (p) \\
\hline \multirow[t]{2}{*}{ MCI } & Single measures & $0.801 \dagger$ & 0.595 & 0.908 & 1.006 & 24 & 22 & 0.497 \\
\hline & Average measures & $0.890 \neq$ & 0.746 & 0.952 & 1.825 & 24 & 23 & 0.078 \\
\hline \multirow[t]{2}{*}{$\mathrm{NC}$} & Single measures & $0.819 \dagger$ & 0.634 & 0.916 & 1.119 & 24 & 24 & 0.393 \\
\hline & Average measures & $0.901 \neq$ & 0.776 & 0.956 & 2.023 & 24 & 24 & 0.045 \\
\hline
\end{tabular}

Table shows results of a two-way mixed effects model in which people effects are random and measures effects are fixed.

*ICCs using an absolute agreement definition.

$\dagger$ Single measure values provide the value for which the estimator is the same, whether the interaction effect is present or not.

$\ddagger$ Average measures values denote an estimated value computed based on the assumption that the interaction effect is absent. 
Table 7: ICC comparison of MoCA 1 and MoCA 3 test scores in MCI and NC patients generated using SPSS

\begin{tabular}{|c|c|c|c|c|c|c|c|c|}
\hline \multicolumn{2}{|c|}{ Group } & \multirow[t]{2}{*}{ ICC* } & \multicolumn{2}{|c|}{$95 \% \mathrm{CI}$} & \multicolumn{4}{|c|}{ F test with true value 0.8} \\
\hline & & & Lower bound & Upper bound & Value & df1 & df 2 & Significance (p) \\
\hline \multirow[t]{2}{*}{ MCI } & Single measures & $0.842 \dagger$ & 0.674 & 0.927 & 1.292 & 24 & 24 & 0.266 \\
\hline & Average measures & $0.914 \neq$ & 0.805 & 0.962 & 2.320 & 24 & 24 & 0.022 \\
\hline \multirow[t]{2}{*}{$\mathrm{NC}$} & Single measures & $0.721 \dagger$ & 0.468 & 0.866 & 0.685 & 24 & 24 & 0.820 \\
\hline & Average measures & $0.838 \neq$ & 0.637 & 0.928 & 1.239 & 24 & 24 & 0.301 \\
\hline
\end{tabular}

Table shows results of a two-way mixed effects model in which people effects are random and measures effects are fixed.

*ICCs using an absolute agreement definition.

$\dagger$ Single measure values provide the value for which the estimator is the same, whether the interaction effect is present or not.

$\ddagger$ Average measures values denote an estimated value computed based on the assumption that the interaction effect is absent.

well as the Area Under the Curve of French Alternate MoCA Tests versus Original French MoCA test for separating MCI from NC.

When MoCA 1 and MoCA 2 scores were assessed, single measure intraclass correlation values for $\mathrm{MCI}(\mathrm{ICC}=0.801)$ and $\mathrm{NC}(\mathrm{ICC}=0.819)$ both fell within their corresponding $95 \%$ confidence intervals $(\mathrm{MCI}=0.595-0.908 ; \mathrm{NC}=0.634-0.916)$ (Table 6).

Similarly, single measure ICC's in the MCI and NC subject population between MoCA 1 and MoCA 3 also fell within their corresponding $95 \%$ confidence intervals. ICC for MCI population was 0.842 (with $95 \%$ confidence interval between $0.674-0.927$ ), whereas $\mathrm{ICC}=0.721$ for the NC population (with $95 \%$ confidence interval between 0.468-0.866) (Table 7).

ICC for MoCA 2 and MoCA 3 in both the MCI $(\mathrm{ICC}=0.784)$ and $\mathrm{NC}(\mathrm{ICC}=0.683)$ cohorts also fell within their respective $95 \%$ confidence intervals $(95 \%$ confidence interval $=0.535-0.902$ for $\mathrm{MCI}$; $95 \%$ confidence interval $=0.399-0.847$ for $\mathrm{NC}$ ) (Table 8).

Thus, given the ICC's observed amongst all three French MoCA tests, within each corresponding subject cohort, the possibility of lack of consistency in terms of reliability and consistency of score measurements due to the fact that a single test administrator was used to distribute all three tests on multiple occurrences, could be ruled out. In other words, all intraclass correlations indicated that the single test administrator used throughout the whole of the validation study was effective in obtaining test scores that were consistent and reliable for all three MoCA test versions in the MCI and NC subject groups.

A receiver operating characteristics plot was also generated to visualize accuracy for MCI detection among the three MoCA test versions. All three versions visually displayed high sensitivity and specificity because there was a trend observed of a vertical incline along with $\mathrm{y}$-axis, with a horizontal incline towards the $\mathrm{x}$-axis (Figure 1).

The area under the curve (AUC) was also calculated for all three receiver operating characteristic curves; the AUC ranged from 0.855 to 0.8885 . The AUC values for all three MoCA versions fell within their corresponding $95 \%$ confidence intervals, which ranged from 0.742 to 0.788 in the lower confidence level, and from $0.969-0.988$ in the upper confidence lever. $\mathrm{P}$ values ( $p=0.000$ for all three MoCA versions) were also calculated (Table 9).

The results from our validation study demonstrated that all three MoCA versions were accurate for detecting MCI positives by displaying specificity and sensitivity (Figure 1). In the case of all three curves, a vertical incline along the $y$-axis towards 1.0 is observed, indicative of high sensitivity in the detection of true positives (MCI positive patients). Additionally, a horizontal trend towards 1.0 along the $x$-axis, representative of specificity was also observed in all three MoCA score curves, indicating the ability to correctly identify the true negatives (non-MCI patients). Finally, accuracy of the three versions of the diagnostic tests was further confirmed by the AUC values, where a trend towards values close to 1.0 was observed (Table 9).

Table 8: ICC comparison of MoCA 2 and MoCA 3 test scores in MCI and NC patients generated using SPSS

\begin{tabular}{|c|c|c|c|c|c|c|c|c|}
\hline \multicolumn{2}{|c|}{ Group } & \multirow[t]{2}{*}{ ICC* } & \multicolumn{2}{|c|}{$95 \% \mathrm{CI}$} & \multicolumn{4}{|c|}{ F test with true value 0.8} \\
\hline & & & Lower bound & Upper bound & Value & df1 & df2 & Significance $(\rho)$ \\
\hline \multirow[t]{2}{*}{ MCI } & Single Measures & $0.784 \dagger$ & 0.535 & 0.902 & 0.915 & 24 & 17 & 0.588 \\
\hline & Average measures & $0.879 \ddagger$ & 0.697 & 0.949 & 1.671 & 24 & 18 & 0.134 \\
\hline \multirow[t]{2}{*}{$\mathrm{NC}$} & Single measures & $0.683 \dagger$ & 0.399 & 0.847 & 0.592 & 24 & 24 & 0.897 \\
\hline & Average measures & $0.812 \ddagger$ & 0.570 & 0.917 & 1.062 & 24 & 24 & 0.441 \\
\hline
\end{tabular}

Table shows results of a two-way mixed effects model in which people effects are random and measures effects are fixed.

*ICCs using an absolute agreement definition.

$\dagger$ Single measure values provide the value for which the estimator is the same, whether the interaction effect is present or not.

$\ddagger$ Average measures values denote an estimated value computed based on the assumption that the interaction effect is absent. 


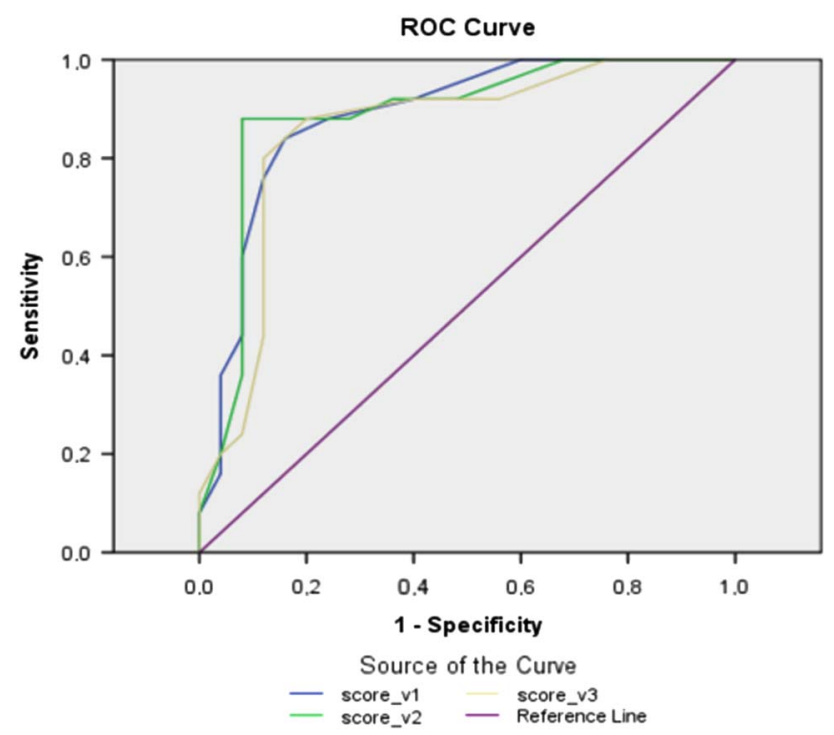

Figure 1: Receiver operating characteristic (ROC) curve for original French versus alternate French MoCA tests. ROC curve displaying sensitivity and specificity of all three MOCA tests where MoCA $1=$ score_v1 (blue curve); MoCA $2=$ score_v2 (green curve); MoCA $3=$ score_v3 (yellow curve). A reference line is displayed as a diagonal line (purple). Positive actual state is 1-MCI. Scores for each version are compiled based on $n=25$ for positive and negative groups.

Hypothesis tests in which p values and confidence intervals were obtained for all three MoCA versions helped further validate the accuracy of each diagnostic test (Table 9). In the case of each MoCA test version, AUCs fell within their corresponding confidence intervals, and $\mathrm{p}$ values demonstrating nonsignificance indicated that all three MOCA versions could be used interchangeably without compromising the accuracy for detection of MCI patients.

\section{CONCLUSIONS}

This validation study shows that alternative French MoCA test versions 2 and 3 are equivalent to the original French MoCA test. The three French MoCA tests versions can be used interchangeably with high reliability and consistency to discriminate between MCI and healthy subjects, thus decreasing potential learning effects when the test is administered frequently.

Table 9: AUC for all three French MoCA test versions

\begin{tabular}{|c|c|c|c|c|c|}
\hline \multirow{2}{*}{$\begin{array}{l}\text { Test result } \\
\text { variable(s) }\end{array}$} & \multirow[b]{2}{*}{ AUC } & \multirow[b]{2}{*}{ SE } & \multirow[b]{2}{*}{$\begin{array}{c}\text { Asymptotic } \\
\text { significance (p) }\end{array}$} & \multicolumn{2}{|c|}{ Asymptotic $95 \%$ CI } \\
\hline & & & & $\begin{array}{l}\text { Lower } \\
\text { bound }\end{array}$ & $\begin{array}{l}\text { Upper } \\
\text { bound }\end{array}$ \\
\hline $\begin{array}{l}\text { MoCA } \\
\quad \text { score_v1 }\end{array}$ & 0.885 & 0.049 & 0.000 & 0.788 & 0.982 \\
\hline $\begin{array}{l}\text { MoCA } \\
\quad \text { score_v2 }\end{array}$ & 0.887 & 0.052 & 0.000 & 0.786 & 0.988 \\
\hline $\begin{array}{l}\text { MoCA } \\
\text { score_v3 }\end{array}$ & 0.855 & 0.058 & 0.000 & 0.742 & 0.969 \\
\hline
\end{tabular}

$\mathrm{p}<0.05$ is indicative of significance. $\mathrm{SE}=$ standard error.

\section{Disclosures}

$\mathrm{ZN}$ has served as a principal investigator and received research grants from Roche, Eli Lilly, V \& V Therapeutics, Eisai, Astra Zeneca, and Merck; and is the director/owner of MoCA Test Inc., receives licensing fees and royalties from licensing agreements when the test is used commercially by pharma, and is the copyright owner of the MoCA test. BBP is a freelance medical writer and has received writing fees from the MoCA Test Inc.

\section{SUPPLEMENTARY MATERIAL}

For supplementary material/s referred to in this article, please visit http://dx.doi.org/10.1017/cjn.2016.273

\section{REFERENCES}

1. Morris JC, Cummings J. Mild cognitive impairment represents early stage Alzheimer's disease. Arch Neurol. 2001;58: 397-405.

2. Petersen RC, Smith GE, Waring SC, et al. Mild cognitive impairment. Arch Neurol. 1999;56:303-8.

3. Petersen RC, Smith GE, Ivnik RJ, et al. Apolipoprotein E status as a predictor of the development of Alzheimer's disease in memory-impaired individuals. JAMA. 1995;273:1274-8.

4. Nasreddine ZS, Phillips NA, Bédirian V, et al. The Montreal Cognitive Assessment, MoCA: a brief screening tool for mild cognitive impairment. J Am Geriatr Soc. 2005;56:695-9.

5. Costa AS, Fimm B, Friesen P, et al. Alternate-form reliability of the Montreal Cognitive Assessment screening test in a clinical setting. Dement Cogn Disord. 2012;33:379-84.

6. Petersen RC. Mild cognitive impairment as a diagnostic entity. J Intern Med. 2004;256:183-94.

7. Müller R, Büttner P. A critical discussion of intraclass correlation coefficients. Stat Med. 1994;13:2465-76.

\section{APPENDIX 1}

Inclusion/Exclusion Criteria for Health Controls Eligibility

$\underline{\text { Inclusions }}$

1. 65 years of age and older

2. 5 years or more of formal education

3. Must be functioning independently in the community

4. Fluent in the French language

Exclusions

1. History of a central nervous system disorder (epilepsy, head trauma, stroke, brain lesion), as well as having any known amnesia or cognitive deficit, or present any condition with susceptibility to causing dementia or cognitive deficit (alcohol abuse, drug abuse, major organ failure, neoplastic disease, sleep apnea)

2. Subject must not have any learning disability, attention deficit disorder, or mental retardation

3. Subject must not have any physical handicap which could influence test results (paralysis, blindness, and severe hearing deficits)

4. Subject must not have preexisting or current major psychiatric illness (major depression requiring hospitalization, bipolar affective disorder, schizophrenia). Previously treated 
depression lacking hospitalization is permitted if subject has been free of any depressive symptoms within the last 6 months and has scored 6 or less on the Geriatric Depression Scale during screening.

5. Subject must not have been on any unstable dose of centrally acting medication in the past 6 months. Stable (for at least 6 months) low doses of hypnotics and antidepressants are allowed.

6. Subject may take antiepileptic medication if it has been prescribed for pain control and NOT epilepsy

7. Subject may take neuroleptics if taken at low doses and as hypnotics

8. Subject must not have a subjective memory scale score of 7 or more
9. Subject must not have above permitted alcohol consumption:

a) 3 drinks or more of $200 \mathrm{ml}$ of $5 \%-7 \%$ alcohol per day for MEN

b) 2 drinks or more of $200 \mathrm{ml}$ of $5 \%-7 \%$ alcohol per day for WOMEN

c) 1 or more drink of $200 \mathrm{~mL}$ of $35 \%$ alcohol per day for MEN and WOMEN

10. Subject must not have consumed drugs in the past 5 years. Occasion marijuana use (maximum once a week) is permitted if not consumed within the last week prior to screening

11. Vitamins, food or herbal supplements, gingko biloba, and omega-3 are allowed. 